\title{
Excellent Online Friendships: An Aristotelian Defense of Social Media
}

Keywords: friendship; social media; Aristotle; privacy; shared activity

\begin{abstract}
I defend social media's potential to support Aristotelian virtue friendship against a variety of objections. I begin with Aristotle's claim that the foundation of the best friendships is a shared life. Friends share the distinctively human and valuable components of their lives, especially reasoning together by sharing conversation and thoughts, and communal engagement in valued activities. Although some have charged that shared living is not possible between friends who interact through digital social media, I argue that social media preserves the relevantly human and valuable portions of life, especially reasoning, play, and exchange of ideas. I then consider several criticisms of social media's potential to host friendships, and refute or weaken the force of these objections, using this conception of a distinctively human shared life. I conclude that we should use the shared life to evaluate features of specific social media and norms for users' conduct.
\end{abstract}

\section{Introduction}

In this paper, I defend the potential for excellent friendship via social media against a number of common objections by appealing to an Aristotelian theory of friendship. I begin by reviewing Aristotle's discussion of the nature of excellent friendship, and applying his conception of the shared life to the activities possible through social media. I argue that excellent, fulfilling friendships may occur via social media, because social media allows friends to share distinctively human activities such as conversation and exchange of thoughts, mutual development of ideas, making art, and playing games. Aristotelians should thus conclude that the best kind of friendship is available online.

I then engage with and respond to six objections: the privacy objection, the superficiality objection, the commercialism objection, the deceptiveness objection, the physicality objection, and the poverty of communication objection. Having addressed these concerns, I explore some ethical issues for friendships online, and conclude by suggesting several ways to use the 
conception of the shared life online to evaluate, design, and use social media so as to facilitate sharing of flourishing lives.

This line of argument is important for two related reasons: first, despite recent skepticism (Cocking and Matthews 2000, McFall 2012, Fröding and Peterson 2012) it shows that friendships conducted predominantly online may qualify as the best sort of friendship. Second, it shows that the online component of friendships taking place both on- and offline (which, according to empirical research, constitute the vast majority of social media users' relationships) is at no significant disadvantage relative to the offline component; online interactions are more than mere stopgaps tiding us over between offline interactions.

Although there are a variety of ways of keeping in touch online besides social media, including chat programs and email, for purposes of this paper, I will focus on social media interactions. Email exchanges look remarkably like pen pal relationships (Briggle 2008) and so may not offer much that is distinctive for the theorist about emerging technologies to be concerned with. Online forums also offer opportunities for online friendship (Kaliarnta et al 2011), but the group dynamics of these forums introduce complications outside the scope of my current project. Social media has drawn a great deal of attention as we attempt to understand technology's impact on modern relationships, and although specific social media platforms vary widely, there is enough distinctive about them for the genre to deserve specific consideration.

People can experience great emotional attachment to online friends, and this alone might seem to be grounds for rejecting arguments that excellent friendships are difficult or impossible online or via social media ${ }^{1}$. But appeals to subjective experience leave open the possibility that such individuals are mistaken about the quality of their friendships; it would be preferable to explain why and how online friendships can be close and fulfilling, as part of a unified theory of

${ }^{1}$ I thank Kai Kimppa for raising this point. 
friendship, and help avoid the charge that people who find online interactions emotionally fulfilling are making some sort of mistake.

\section{The Shared Life Online}

Aristotle divides friendships into those based on usefulness (utility friendships), pleasure in each other's company (pleasure friendships), and valuing each other as good in themselves (virtue friendships). Virtue friendships are the best form of friendship, but take the most time to develop, and are the most rare — because, as Aristotle points out, good people are relatively rare, compared to people we might find useful or pleasant.

This does not mean that virtue friends are useless or unpleasant. Good friends are good to each other. Furthermore, many virtue friendships begin as lesser friendships. It takes time and repeated interactions for people to become familiar with each other's characters. The lesser forms of friendship make excellent opportunities to become better acquainted with people. What distinguishes the highest form is not whether or not a friend is pleasant or useful, but whether these traits exhaust the value of the relationship.

Virtue friends have special value, on Aristotle's account, because they share their lives with each other, and in doing so, each becomes "another self" to the other. (NE 1166a30, 1170b5, 1171b30-35) By expanding a sense of self beyond one's immediate concerns, a person can enjoy more good life than is realizable alone. (NE1170a-b) The shared life is thus crucial to an Aristotelian theory of the best form of friendship.

In one sense, social media and other forms of online interaction seem antithetical to shared living, because they do not (or need not) involve physical cohabitation — one can have online interactions with a person across the globe as easily as the person in the next room. But in explaining what he means by the shared life, Aristotle clarifies that for human beings, living 
together is accomplished by sharing "conversation and thought," not "grazing in the same field, like cattle." (NE, 1170b10-15) Any medium allowing friends to share conversations and thoughts should thus be compatible with virtue friendship.

The best form of friendship is found between people who share both the broadly human good of rationality, by engaging in collective reasoning through conversation, as well as more specific goods valued by the particular friends:

... whatever his existence means to each partner individually or whatever is the purpose that makes his life desirable, he wishes to pursue it together with his friends. That is why some friends drink together or play dice together while others go in for sports together and hunt together, or join in the study of philosophy: whatever each group of people loves most in life, in that activity they spend their days together. For since they wish to live together with their friends, they follow and share in those pursuits which, they think, constitute their life together (NE 1172a1-7).

The shared life must, then, include both sharing of broadly human good, and of particular goods for particular human beings. It is in virtue of this rich and personalized sense of a shared life that we ought to understand friends as other selves.

The shared life in the relevant sense cannot plausibly mean living together as physical cohabitation, hence the caution about differentiating 'living together' in the friendly sense from 'living together' in the cattle sense. Friends, rather, 'live together' by sharing rational life, by sharing language and thought through conversation, as the rational life is characteristic of human beings and conversation is how we reason collectively. In addition, different people find meaning and value in their lives by pursuing different types of personal projects. Individuals share lives with their friends by sharing these activities together: by drinking together, gambling together, working out or playing sports together, hunting together, philosophizing together. In such activities, for virtue friends, a friend is not merely valued as means to an externally defined end. The workout partner is not valued merely as pleasant company on runs, the fellow 
philosopher is not valued merely for providing feedback on drafts in progress and helping work out ideas. Rather, she is perceived by the friend - and the friend reciprocates, perceiving her in turn - to be sharing in the valued activity and the valuing, thus expanding each friend's capacity for enjoying worthwhile activities.

In evaluating the potential for friendship over social media, then, we have to look at the sorts of conversations and thoughts exchangeable in this medium, as well as the sorts of activities which could plausibly be shared online. Can philosophically-minded friends discuss philosophy using social media? Can photography enthusiasts share and critique each other's photos? Can gamers play together online? Recall that, even for activities which are more difficult to share online, it can still plausibly be important to individuals who value such activities to reason about them, as reasoning is a characteristically human activity, and conversing about other activities allows people to reason about them together. Sharing a conversation about one's day with a friend should count as living together, for Aristotelians, if we are to take his comments on the nature of the shared life seriously. Friends need not be present for every life event in order to share in a life: they needn't be grazing in the same field, like cattle. Our capacity to share our lives and thoughts via language and other symbolic representation and artistic expression surely plays a role in the kind of sharing of ideas, experiences, and perceptions that constitutes the relevant sense of living together, and conversations are facilitated rather than discouraged by many social media. We should thus conclude that virtue friends may use social media to share their lives.

Another popular use of social media is to share pictures, photographs, music, and video footage. Owing to technological considerations, many of these artifacts were not, for Aristotle, goods that he could have imagined people sharing, at least without directly sharing space. In 
ancient Athens, friends would have needed to attend the same play at the same time in order to enjoy it together, or listen to the same musical performance. But with today's recording technology, in conjunction with the capacity to share and exchange recordings, photographs, and so forth, friends can experience the same events from different times and places, as part of their friendship: a friend might send me a link to a music video for a song he enjoys and thinks I would like to hear as well, or we can both watch the same movie, even though we are in different parts of the world. Sharing such a song can be a way for us to expand our mutual capacity for enjoying good music; if I am confident that my friend values music as I do, I will find it intrinsically valuable that she listens to good music. The popularity of social media for exchanging links to movies, music, and online art suggests that we find it important to share these experiences with others via nonverbal means not imagined by Aristotle. However, sharing such goods in this way seems consistent with the spirit of his characterization of living together as sharing human activities: sending a friend a link to a song, for example, strikes me as a human, not a cattle-ish way of sharing the experience of listening to music we both find valuable and enjoyable.

Yet another common use of social media is as a space to play games. Online gaming is a major way that people play games together, and is valued, among other things, as a way of maintaining social relationships (Wohn et al 2011), so online gaming looks like another plausible candidate for the shared life (Munn 2012). Games are the basis of numerous friendships and playing games together is a shared activity which many people think gives their lives meaning. It engages many of our characteristically human capabilities; playfulness, strategic reasoning, creativity, and exploration. Many online gaming relationships will be instrumental. People might be friends online merely as a means to greater personal success at the game, or for the pleasure 
of playing together without necessarily valuing the other person's gaming as the activity of one's 'other self'. But it is not what friends do, but how they value what they do, that makes the crucial difference for distinguishing virtue friendship from the lesser forms. Although some friendships between players of online games will thus be merely instrumental friendships - friendships of pleasure or utility - others can be virtue friendships, provided friends value each other's lives and activities in the right way.

\section{Objections Considered and Refuted}

While people rarely think that virtue friends must physically cohabitate, many doubt that excellent friendship can be realized online. If these criticisms were correct, they would cast doubt on my claim that friends can plausibly "live together" online.

If friends cannot trust each other online, or cannot share their valued activities owing to a lack of privacy, cannot interact with each other as particular individuals rather than through mere superficial broadcasting of interests and life events, or if their relationship is adversely affected by the commercialism of social media, or if the best friendship involves a kind of physicality impossible to experience in online interaction, or if the communication available online is impoverished so that conversations online do not allow friends to enjoy sufficiently rich exchange of ideas to allow friendship to flourish, then my argument will have been insufficient to show that social media supports excellent friendship.

I therefore consider and refute six objections to my thesis: the privacy objection, the superficiality objection, the commercialism objection, the deceptiveness objection, the physicality objection, and the poverty of communication objection.

\subsection{Privacy Objection}

Because social media facilitates highly public conversations, the intimacy characteristic of 
the best friendships would seem impossible on most common social media sites. Friendships cannot thrive without the capacity to confide secrets (Thomas 1987). Otherwise-friendly exchanges on, for example, one's Facebook wall seem much too public to allow for such intimacy. Even if one's privacy settings are relatively locked down, the exchange would still be visible to all of one's other contacts.

My response to this objection is two-pronged. First, many social media include one-on-one communication channels as well as broadcasting platforms. So most social media developers already recognize the importance of private communication channels. This may not be much consolation, however, on its own, as one might think this still leaves the most distinctive parts of social media - those things which set it apart from older technologies such as email - inimical to friendship.

However, the second portion of my response is to point out that not every interaction between friends plausibly involves secrets. Conversations conducted publicly may fail to allow for the exchange of secrets, and even convey an implicit invitation for other interested parties to join in, and simultaneously constitute important parts of a friendship. Friends can socialize at parties, making their conversations implicitly open to other party-goers, and such conversations may constitute rich and fulfilling sharing of lives between friends.

Secrecy may be necessary for friendship in the long run, but it does not follow that every exchange in a friendship must be conducted in secrecy, nor that the public parts of a friendship are any less important to the friends or the wellbeing of the friendship. Unless we rule out social and semi-public settings more generally as appropriate grounds for friends to interact, we cannot consistently criticize social media on this basis, and the more general criticism seems highly implausible. 


\subsection{Superficiality Objection}

According to the superficiality objection, on social media one simultaneously addresses everyone one knows, and no one in particular, and so such exchanges are superficial; rich conversation with particular friends, of the sort which would constitute sharing a life, is difficult or unlikely (Bloomfield, forthcoming). More directed address of particular friends would call on and invite their unique perspectives and contributions, but a broadcast to all one's acquaintances online is necessarily so thin and watered-down that the richness of personalized exchange is lost.

My response is that this presumes one (perhaps common) way of using social media as the option, or at least the default option. But if something is wrong with the broadcasting approach, and I am sympathetic to concerns to this effect, this at best constitutes a criticism of people's use of the medium, not the medium itself.

A better model for online exchange seems to be something like the following: Public postings may be directed at friends with a shared interest, even while conveying an implicit invitation for others to join in the conversation. These directed addresses may be quite specifically tailored to call on a friend's shared interests, character, sense of humor, and so forth, and simultaneously convey an implicit invitation for the like-minded to join in. Although public, links, stories, pictures, and videos can be shared because one believes a particular friend would like them, find them interesting, want to respond to them, and so forth, and the friend could plausibly accept these friendly tokens without finding them diminished for being communicated in a public forum.

Suppose, for example, that I post pictures of a recipe in progress to my Facebook feed, so that a couple of my friends who share my interest in cooking can contribute suggestions, answer questions ("Help! Why is this dough taking so long to rise?"), and share in the experience. (Some 
of these friendships may be instrumental, but if cooking is valued by the friends in the right way, sharing my cooking experience with you may be part of our shared life and hence part of a virtue friendship.)

Because this conversation is public, another acquaintance may thereby realize we share a hobby and join the discussion. But it is not obvious that this cheapens or detracts from conversation with my established fellow cooking enthusiasts, any more than would an equivalent conversation at a public gathering where the acquaintance joins in.

Thus, social media poses no intrinsic threat to the richness and particularity of friendly interactions. There may be some temptation toward making superficial broadcasts online, but there are alternatives available. At best, we end up with a normative claim about how users ought to interact via social media, rather than a condemnation of the medium. Users will be best positioned to enjoy excellent friendships online if they show appropriate sensitivity to social context and some skill at handling complex social exchanges, but this is not so different from the skill set which benefits any other friendship.

\subsection{Commercialization Objection}

A number of prominent social media platforms are commercial in nature. Companies such as Facebook and Twitter make money off of their users in a variety of ways. Most prominently, purveyers of free-to-users networks tend to make their money through advertising: the users are, at the end of the day, the product (a dedicated audience, about whom the company knows quite a bit of information which advertisers find valuable as a means to more effectively target their market), being sold to advertisers who are the ultimate consumers of such networks. Users of commercial social media, arguably, ought to know and take into consideration this arrangement, but one still might wonder whether friendships conducted in such environments are ethically 
problematic - are friendships somehow weakened, harmed, or made less valuable if they are the means by which the companies providing the space earn profits?

In order to effectively address this challenge, it is helpful to distinguish several potential problems commercial social media might pose for friendship. It might be the case that friendships conducted under such circumstances, for example, are qualitatively inferior to those in which the friends do not serve as sources of revenue in virtue of their friendship - in something like the way that "diet" versions of food are often qualitatively inferior to those made with plenty of butter and sugar. It might be that friendships on social media are at no intrinsic disadvantage, but that companies which profit from such friendships tend to harm the friendships in other ways, perhaps by snooping, violating privacy and interfering with communication channels, as when Facebook changes the items displayed in its News Feed (a non-exhaustive display of updates from contacts on one's "Friends" list). It could be the case that friends harm each other by encouraging their friends to serve as products for such companies to sell. Or, upon closer examination, the problem of commercialism might not look so terrible after all.

Tackling these in order: Consider the first challenge: that friendships on commercial social media are weaker or less valuable than their equivalents elsewhere. It is implausible to me that friendships and friends' interactions are intrinsically qualitatively less when and because other people profit from them. If I go to the bar with my friends on Trivia Night, the bartender and bar owner benefit from my friendship, but it is implausible that this weakens or lessens the friendship.

Someone might object that this is disanalogous, because friends at a bar are not the product; they are the consumers. But other scenarios can be constructed in which friends engage in activities from which others profit, even ones in which the friends are products rather than 
consumers, and where friendships do not thereby seem weakened. Suppose my friend and I together perform at a local coffee shop's open mic night. The coffee shop owner holds such events because they attract paying customers, even if the performers purchase nothing: they are the product being used to sell the coffee shop and its related goods to other consumers. And yet writing songs and rehearsing them, with the goal of eventually performing them at an open mic, does not seem intrinsically less friendly than the aim of writing and performing them for some other end.

Or, consider the practice of holding Ladies Night at bars: in this business model, the establishment offers free drinks to women, in the hopes of attracting more male clientele, said men being attracted by the opportunity to make the acquaintance of the women attracted by the free drinks. If a woman and her friends decide to take advantage of Ladies Night as an opportunity to socialize, they do not seem to be at any particular disadvantage, at least so far as their friendship goes. (They may or may not advance their other interests, depending upon their tastes, preferences, and desires, as well as the conduct of the other patrons of the bar.) In this case, the friendship does not seem to be threatened or lessened in virtue of the bar owner profiting from their use of the goods offered in hopes of attracting women to the bar, in order to sell goods to someone else. So I conclude that it is implausible that friendships on commercial social media platforms are intrinsically lesser than their nonprofit counterparts.

I take much more seriously the charge that friendships can be harmed by violations of privacy, and the trust friends place in each other. It is highly plausible to me that one of the bad things about, for example, government surveillance is the damage it does to friendship. But there is an important disanalogy between state surveillance and the data monitoring in which commercial social media companies engage; the voluntary nature of friends' involvement. If the 
government eavesdrops on you, they do so without your consent, and possibly without your knowledge. If friends are aware that their conversations and exchanges may be monitored, have other options (such as email and noncommercial social media networks) for interacting, and yet choose to engage with each other in this setting, fully conscious of the circumstances, that seems to me no bigger a threat to their friendship than having a conversation in a public space where others can eavesdrop... such as at a bar, coffee shop, restaurant, or public park. If social media providers engage in deception about the type or scope of their information gathering and monitoring practices, so friends do not make their choices in full knowledge of their lack of privacy, then I think the company does wrong - and there seems to be evidence that companies do engage in such deception on occasion. But I do not think this need be the case in commercial social media, and it is important to distinguish specific harms of wrongful action from generalized threats intrinsic to the medium.

The last possibility also seems to me to be defused by emphasizing the voluntariness of participation in commercial social media. Friends may choose to interact via social media, but friends who try to force others to do so do wrong -not because of something about social media, but for failing to respect their friends' agency, for attempting to coerce them, and for failing to take seriously their friends' reasons for preferring not to engage. Note that the last is distinct from concerns about whether these friends are correct in preferring not to use any particular form of social media - as I have argued elsewhere (Elder 2014), good friendship seems to require some willingness to treat the friend's subjective interests as valuable, simply in virtue of their being the friend's, and so friends who fail to treat their friends' subjective interests as valuable are thereby failing at being good friends.

Because so many of my responses appeal to voluntariness, it is entirely possible that those 
traditionally considered incapable of giving voluntary consent, particularly children and youths, may not be well-suited to make good decisions about their social media usage, and this may have impacts on what children should be permitted to agree to - or what companies can do with their underage user base. ${ }^{2}$ But at minimum, adult friendships conducted on commercial social media emerge relatively unscathed.

\subsection{Deceptiveness Objection}

It is easy to make deceptive claims about oneself online, either deliberately or by subconsciously editing what one reveals about oneself. (Walther 2007, Hancock 2007) Therefore, runs the deceptiveness objection, trust in online friends should be minimal, or if one trusts one's friends, such trust is ill founded. A friendship grounded in ill-founded trust seems to be a weaker or lesser friendship than one where trust is merited, one might suppose, and so online friendships are bound to be weaker or lesser than their off-line counterparts.

But we must be careful to consider the alternatives: in this case, the problem of deception in face-to-face interactions, and the potential for offline friendship despite such risk. Deception and editing of the self one presents to acquaintances is quite possible - and commonplace! - in person, and face-to-face interactions also present vulnerabilities. Skilled performers and con artists, for instance, exploit people's confidence in their ability to gauge character by expression and tone of voice, so the "mark" fails to pay adequate attention to the content of the con artist's claims. Even in more ordinary and less sinister exchanges, we frequently use voice, tone, expression, and posture to elicit desired responses from our conversation partners and to subtly direct the conversation toward topics we enjoy and away from uncomfortable subjects.

Text-based conversations are, arguably, less susceptible to deception of this kind: one can

\footnotetext{
2 The documentary Generation Like (Rushkoff, D. (Director), Public Broadcasting Service, 2014) vividly portrays some of the problematic situations that can arise between underage users and commercial social media providers.
} 
evaluate the content and consistency of claims without the emotional overlay introduced by facial expressions and tone of voice. Online, conversations leave digital "paper trails", making it easier to cross-check stories and consider a person's comments in light of the overall picture of their character presented by their online presence. For example, the person who expresses one view on social issues to you, but whose Facebook wall is full of posts and memes to the contrary, gives grounds for an overall assessment of character which takes the totality of evidence into consideration.

It is by no means clear that the grounds for trust online versus in person weigh heavily in favor of one versus the other, and there is good reason to think one ought to exercise good judgment and discrimination in both circumstances. Even if deception is easier or more common online, at best this is a difference of degree, not kind. There is also evidence that people tend to detect quite a bit about online correspondents' intentions and truthfulness via things like word choice and phrasing, which will tend to help mitigate concerns about the ease with which ordinary people can deceive each other online. (Wallace 1999) If the potential for deception in real life is not sufficient to rule out the possibility of friendship, neither should it be considered especially hazardous to online friendship.

\subsection{Physicality in Friendship}

One of the most obvious differences between friendships online versus off is the possibility - or lack thereof - for physical contact, and more generally, the physical presence of the other person. As social creatures, many of our social needs may be met through online interaction but some may not be. In many contemporary Western cultures, particularly between men, physical touch is not typically recognized as an important component of friendship and is often considered taboo (Strickwerda and May 1992, Derlega et al 1989, Monsour 1992, Bank and 
Hansford 2005), but for many people touch can be an important and valuable part of sociality. Furthermore, just being around other people can itself be important to many of us; think about the attraction, for some people, of heading out to a coffee shop to read rather than staying at home, or working on a project at the library rather than in a solitary office.

Clearly, these social goods cannot be realized in online friendships, while they are often part of offline friendships, although with the caveat that this will vary depending on the individual and the culture. In Arab cultures, for example, male friends often hold hands, while such behavior is considered highly unorthodox in the US: see, for example, the furor over a US president holding hands with the king of Saudi Arabia (http://www.cbsnews.com/news/abdullahbush-stroll-strikes-nerve/). This raises questions about whether members of less physically affectionate cultures thereby miss out on valuable, and it seems plausible to me that the answer could be "yes".

Does this mean that online friendships are intrinsically at a disadvantage relative to offline ones? Perhaps, but I am not sure that this disadvantage need be severe. In sexual relationships, many (although not all) people find monogamy important, but there is no parallel norm for friendship; in fact, it is often thought to be healthy for people to have different friends who meet different needs, share different interests, and serve as sources of very different goods; in fact, variety in friendship may plausibly be one component of an excellent life. Given this, it is not an easy or obvious transition from the thought that one social good is unavailable in a particular friendship, to the conclusion that the friendship is thereby deficient, nor that one is worse off for having that friendship. So long as one's need for physical contact and sharing space is met (and such needs may vary widely depending upon the individual in question) some particular friend need not be a source of those goods. What may be the case is that people are worse off if none of 
their friendships involve physical presence or physical contact, but unless online friendships replace offline ones in a person's life, online friendships do not thereby make people worse off, and the data so far suggests that online friendships do not replace offline ones; rather, they take place during time that would otherwise have been spent on solitary activities, such as reading or watching television [cite Vallor's source here: Wellman et al. 2001; Ellison et al. 2007;

Rainie et al. 2006; Ito et al. 2008].

\subsection{Poverty of Communication Objection}

The final objection I consider is what I will call the poverty-of-communication objection. Cocking \& Matthews (2000), Vallor (2012) and McFall (2012) introduce related objections to the potential for the best form of friendship online. Although Cocking \& Matthews' original concerns were addressed in Briggle (2008), later authors have not found this response entirely satisfactory (Vallor 2012, McFall 2012). I supplement Briggle's account by introducing a distinction between sharing information about activities, and sharing the activity of discussing or reasoning about that same information. Making this distinction helps address concerns about the feasibility of sharing lives online.

Cocking \& Matthews (2000) and McFall (2012) (and, to some degree, Vallor 2012) all criticize the nature of online communication. They express concern that something about the nature of communication online makes it impossible, or at best, significantly more difficult or unlikely, for friends to share thoughts and feelings in ways constituting an Aristotelian shared life. This is related to both the deceptiveness and superficiality objections addressed earlier, but is raised often enough to deserve a dedicated response.

Friends may converse about their lives online, they charge (it would be difficult to deny that friends do, at this point in time), but such conversation fails to count as the rich sort of 
sharing Aristotle had in mind.

This sort of objection can involve either of two claims: 1) I cannot actually share activities with my friends, only talk about my activities with my friends; 2) Even if such talk constitutes a shared human activity sufficient for Aristotle's sense of the shared life among friends, such talk is in principle impoverished when conducted via text as opposed to a face-to-face conversation and so is an inferior part of a friendship. For example, in relating something to a friend face-toface, my expression might give me away at a key point in describing an emotional encounter, but textual communication strips away such supplementary information channels. Online, I merely report my conscious experience and interpretation of my life to someone who can then contribute little to my understanding of the portions of my life for which my friend is not present and of which I was not initially aware. (Cocking and Matthews 2000)

I think that neither claim is warranted. Consider the first one first. Vallor (2012) expresses it as follows:

...the possibilities for sharing lives online look relatively impoverished if we grasp the distinction between sharing lives and sharing about lives; the former involves performing together the activities that make up a life, the latter involves communicating to one another information concerning our lives, without implying shared activity. (Vallor 2012, 196)

But this does not hold up under scrutiny.

Multiplayer gaming is perhaps the most obvious example of a shared activity that is plausibly conducted online and which could constitute the sort of shared life characteristic of virtue friendship (Munn 2012). Other examples, however, come readily to hand. Journaling has a longstanding foothold in social media, distinguished from private diary-keeping by the fact that such journals are kept with the intention of introspecting on one's life in the company of others. (Soraker 2012; Briggle 2008) 
Events described in a journal entry are not directly shared with the friends, any more than the experiences relayed during a conversation transport one's conversational partner to the scene described. However, the activity shared by participants in journaling is not the event related in the journal entry, but the journaling. Journaling is a respectable activity in its own right, valuable for introspecting and reflecting on oneself. It is useful for gaining self-knowledge as well as better control over the direction of one's future.

Writing, reading about, and discussing one's life is something that can be shared with friends and to which friends can be unique contributors, offering valuable insight on something one might not have noted on one's own. Having a co-perceiver of one's perceptions can greatly enhance one's understanding and development of oneself. Rather than think public journaling consists merely of relaying one's day as one experiences it, to which friends and observers could contribute nothing new, as charged in the objection, journaling is plausibly valuable precisely because narrating one's experiences in order to reflect on them can offer people new insights into themselves and each other.

This use of social media is directly related to character development of the kind Aristotle specifically calls out as characteristic of the best friendships: both appreciation of extant character and the role played by friends in modeling, critiquing and offering feedback which shapes the development of a friend's character.

Not all friends engage in shared journaling, of course, and other activities may be harder to share online; friends who hike together, for example, may find shared journaling about hikes to be a poor substitute for actually climbing a mountain together. But that doesn't show that shared activities online are inferior for friends who find online activities - such as gaming and journaling - to be intrinsically rewarding. For comparison, consider again the friends who hike; 
the fact that they find shared hikes to be an important component of their friendship does not mean that all friends must hike. Conversely, we can easily imagine that another group of friends (perhaps a group of gamers) might find hiking to be unpleasant and, at best, instrumentally valuable for maintaining cardiovascular health and only made bearable by the fact that on a shared hike, the friends can discuss gaming, even though they consider such discussions a poor substitute for actually gaming together.

Conversations, moreover, may be directly shared online. Conversationalists do not directly share their described experiences with conversational partners, but the conversation itself is a direct activity in which both friends engage, and to the extent that conversing is collective reasoning and reflection on one's life, friends thereby share lives. I suspect this is Aristotle's point to begin with, in distinguishing human "living together" from cattle "living together". In that case, textual communication is just another way (and, in addition, a distinctively human way) to have such conversations.

This brings me back to the second aspect of the poverty-of-communication objection. Many nonverbal cues are present for face-to-face exchanges but missing in textual communication. These nonverbal cues are lauded by McFall (2012) and Cocking and Matthews (2000) for being "involuntary" and "unconscious" and thus (presumably) superior indicators of one's true self. But as noted in the previous section, we often exaggerate or consciously control facial expression or tone of voice for dramatic effect. On the other hand, word choice and phrasing can reveal quite a bit about a person without their consciously intending to do so. (Søraker 2012; Wallace 1999) Furthermore, Briggle (2008) makes a persuasive case that online communication, relative to face-to-face discussion, enhances important qualities such as candidness and deliberateness. 
Thus, conversing online still counts as a directly shared activity, and one in which the sensitive and skilled writer and reader can participate fruitfully. Of course, not every social media user is sensitive and skilled, but neither is every conversationalist - one can be tone deaf, oblivious, and so forth, and thereby have one's potential for friendship limited by such handicaps. Meanwhile, generations of prose testify to the potential for rich communication by text. Skills of writing and reading then turn out to be important for developing and maintaining rich social relationships online, just as the ability to be both a good speaker and a good listener are important to developing and maintaining good face-to-face friendships. They help us to excel at the activities that constitute friends' shared life.

\section{Aristotelian Guidelines for Online Friendship}

It is not, of course, my intention to argue that all friendships online are good ones, or even that all close online friendships are good. In what follows, I explore some ways that friends' actions can badly impact online friendships.

\subsection{When Bad People Team Up Online}

Aristotle's account of virtue friendship makes virtuous character central to the best friendship, and holds that people who are close but not virtuous can be actively bad for each other, especially by sharing vicious activities with each other. “...the friendship of base people turns out to be vicious," Aristotle argues, "For they [the friends] are unstable, and share base pursuits; and by becoming similar to each other, they grow vicious." (NE 153/ 1172a10) As I have argued elsewhere (Elder 2014), friends with bad character can be bad for each other in a variety of ways, by sharing bad activities (that is, living badly) together, by influencing each other for the worse, and by reinforcing existing bad tendencies and thus making it more difficult or less likely for their friends to improve. Here I focus on ways that this is specifically important 
in online friendships, especially those conducted via social media.

As we have seen, Aristotle thinks the shared life is central to friendship: the quality of the life shared determines the quality of both the friends and the friendship. One's quality of life is at least heavily influenced by one's character, particularly the activities in which one engages and which substantially constitute one's life. The good life (eudaimonia) is the life of the virtuous person who has the external goods necessary for such a life, and friends who enjoy the best sort of friendship will share the best sort of life. Given the variety of artistic and intellectual pursuits which can be pursued online, as well as pleasant components of a good life such as games and entertaining and enjoyable conversations, it is plausible to think that many excellent activities can be shared online and so help constitute excellent online friendships.

But of course, not all online activities are excellent, and some are characteristic pursuits of the vicious rather than the virtuous; friends who share in such activities would be close but not good for each other, on Aristotle's account. Even though they share lives, the life they share is not worthwhile: "What is good by nature is also good for the decent person; that is why life would seem to be pleasant for everyone. But we must not consider a life that is vicious and corrupted, or filled with pains; for such a life lacks definite order, just as its proper features do." (NE 149/1170a20) Taking this in conjunction with the prior quote about base people sharing bad activities which would not be part of a good life, we end up with the idea that friends must share good lives in order to be the best of friends. While there is not space here to do a proper defense of this feature of eudaimonist virtue ethics, it is central enough to Aristotle's theory that it follows quite naturally to maintain that friends who engage in vicious activities online, whether bullying, plotting terrorist activities, or the unethical forms of hacking, do not live the good life 
together and so are not good friends, even though they may be very close. ${ }^{3}$ (See Elder 2014 for a fuller discussion of how bad people can be close friends without being good friends.)

More subtly, but just as importantly, many of the common elements of online friendship, such as journaling, conversations, and discussions of various forms, have friends both revealing their own characters and influencing each other, both subtly and directly: from explicit advice and prescriptions through modeling and emulating each other, to reinforcing each other's vices or virtues in discussions and shared deliberations. Friends may thus have great influence on each other's characters in online (and offline) interactions, and if character is important to one's ability to live the good life, as eudaimonist Aristotelian virtue theorists hold, then good friends will influence each other in good ways, while bad people will do the opposite and thus be bad friends, whether or not they intend to do so.

\subsection{Pressuring Friends to Join or Use Social Media}

Friends sometimes feel pressured to join or use social media to keep up with their friends. ${ }^{4}$ They may even find themselves left out of invitations and event planning conducted via social media to which they do not attend. Although such social dynamics are no doubt real and problematic, this does not seem to pose any special challenge to social media in particular; friends who share the same space and communication channels are often at an advantage relative to friends with whom one has to go out of one's way to connect.

Good friendships seem as though they can be sustained despite such obstacles, and while I argue here that good friendships are possible via social media, this is not meant to condone attempts to restrict friendly activities to such media when it is inconvenient or otherwise unattractive for one's existing friends to do so. But the problems with the sort of behavior

\footnotetext{
3 Thanks to Olli Heimo for raising this question.

4 Thanks to Erica Neely for raising issues about relationship dynamics online, which inspired this and the following section.
} 
described here do not seem to be the result of social media per se, but rather implicate broader issues about friendliness, cliqueishness, inclusivity, and other social challenges with which the would-be friend must wrestle, regardless of technology. It seems intuitively unfriendly to insist, for example, that one's friend accompany a person to an expensive restaurant when the friend is trying to save money, but this does not show that there is something wrong with expensive restaurants; rather, it demonstrates that good friends ought to be sensitive to their friends' limitations and other interests, and work to find a common ground.

That said, if social media is an easy and convenient way to keep in touch, it is plausible that in some circumstances the friend who refuses to do so is in the wrong, much as the friend who refused to use phone calls to keep in touch and insisted on making all interactions take place “in person" might, under some circumstances (and here the details would definitely make a difference) be "difficult" in an unfriendly way and place unreasonable demands on the friendship.

\section{3 "Blocking" others on social media}

Relatedly, some relationships online become strained when people confuse standards for broadcast-type communication with issues of personal communication in friendship. Social media programs often give users the ability to block material originating from users whose content they wish to avoid. Although such behavior seems unlikely in characteristically close and high-quality friendship, it can be a helpful way to manage one's contact with looser social connections whose tastes, interests, and politics clash with one's own.

Relational friction can transpire when someone discovers that a connection has blocked their feed. To make sense of these cases, it is important to differentiate kinds of concerns. From a political perspective, when one person blocks another from their news feed, no harm is inflicted 
on either party: freedom of speech is standardly interpreted as not entailing that others must listen to you, and so exercising discretion in what one chooses to consume on social media does not, plausibly, infringe on others' rights or violate any legal duties or obligations. "Friends" who object to being blocked by claiming something like a violation of their freedom of speech thus miss the point of the principle to which they appeal. However, in close interpersonal relationships, such practices can be used to minimize conflict, sometimes at the cost of honesty and openness. It can be cowardly to avoid difficult conversations with a person, simply by refusing to look at the things they say or do with which you disagree strongly.

However, neither are the blocked parties necessarily blameless; failure to take one's audience into consideration when (for example) endorsing something that belittles or demeans people with whom one wishes to maintain a close relationship seems ill-advised and unfriendly. Deciding whether a comment calls for tactful avoidance or honest and open airing of grievances can be highly difficult and calls for skill and sensitivity to a variety of concerns, including how close one is or desires to be to the other person. This, in turn, can itself be challenging; the boundaries between close friend and loose acquaintance are often blurry in real life, even as we acknowledge differing norms for each kind of relationship. It remains the case, however, that good friends can and should be able to overcome such difficulties without taxing an Aristotelian theory of friendship, nor the resources of social media. Instead, characteristics such as tact, forthrightness, and sensitivity to context, courage to be able say the difficult thing, and wisdom to know which fights are worth having and which are not, remain relevant today as before. What distinguishes the virtuous person is not so much which tools they use, but when and how they choose to deploy them.

\section{Conclusions about Friendship and Social Media}


Many social media friendships will of course be superficial, and likewise many online exchanges: social media is useful for keeping in touch with casual acquaintances. But the majority of offline friendships are also utility and pleasure friendships. Aristotle tells us the best kind of friendship is rare (NE 1156b25). But there is no principled reason to think that it couldn't be carried out online, and reason to think social media offers another way to realize a difficult-toobtain but valuable good.

We should draw several lessons about desirable features of social media and wise, prudent ways to conduct our friendships online from what we have seen here. The range of conversations possible in a flexible medium like Facebook may better facilitate sharing lives than a highly constrained platform such as Instagram or Twitter. If privacy is important to friendship, trustworthy and user-friendly private messaging systems may be important for friendshipconducive social media. Options for controlling how narrowly or broadly one's message is distributed, by grouping friends into "circles," as on Google Plus, may prove important ... or perhaps not. The serendipity of unexpected connection might be part of social media's charm. Treating posts as implicit invitations to converse, rather than broadcast announcements, may be necessary to realize certain kinds of social goods. The ability to objectively but sympathetically evaluate people based on what they say and do online turns out to be a valuable skill to cultivate.

Rather than fear social media as a threat to genuine friendship, we should consider how it can be used to foster an important good, by considering it in the context of the shared life characteristic of the best friendships.

\section{Acknowledgements}

TK

\section{References}


Aristotle. Nicomachean Ethics. $2^{\text {nd }}$ ed. (1999). Translated by Terence Irwin. Hackett.

Bloomfield, P. (forthcoming). Friendship on Facebook. In Social Media and Living Well, eds. B. Beasley and M. Haney. Lexington Press.

Briggle, A. (2008). Real friends: How the internet can foster friendship. Ethics and Information Technology 10(1), 71-79.

Cocking, D. \& Matthews. S. (2000). Unreal friends. Ethics and Information Technology 2(4), 223-231

McFall, M.T. (2012). Real character-friends: Aristotelian friendship, living together, and technology. Ethics and Information Technology 14(3), 221-230.

Munn, N.J. (2012). The reality of friendship within immersive virtual worlds. Ethics and Information Technology 14(1), 1-10.

Ellison, N. B., Steinfeld, C., \& Lampe, C. (2007). The benefits of Facebook "friends": Social capital and college students' use of online social network sites. Journal of ComputerMediated Communication, 12(4), article 1. http://jcmc.indiana.edu/vol12/issue4/ellison.html.

Fröding, B. \& Peterson, M. (2012). Why virtual friendship is no genuine friendship. Ethics and Information Technology 14(3), 201-207.

Ito, M., Horst, H. A., Bittanti, M., Boyd, D., Herr-Stephenson, B., Lange, P. G., Pascoe, C. J., \& Robinson, L. (2008). Living and learning with new media: Summary of findings from the digital youth project. http://digitalyouth.ischool.berkeley.edu/report.

Kaliarnta Sofia, Nihlén-Fahlquist Jessica, Roeser Sabine. (2011). Emotions and Ethical Considerations of Women Undergoing IVF-Treatments, HEC Forum, 23, 281-293

Rainie, L., Horrigan, J., Wellman, B., \& Boase, J. (2006). The strength of Internet ties. Pew Internet and American Life Project, http://www.pewinternet.org/Reports/2006/TheStrength-of-Internet-Ties.aspx.

Thomas, L. Friendship. Synthese 72(198), 217-36.

Vallor, S. (2012). Flourishing on Facebook: Virtue friendship \& new social media. Ethics and Information Technology 14(3), 185-199

Wallace, P. (1999). The psychology of the internet. Cambridge University Press.

Søraker, J.H. (2012). How shall i compare thee? Comparing the prudential value of actual virtual friendship. Ethics and Information Technology 14(3), 209-219.

Wohn, D.Y.; Lampe, C.; Wash, R.; Ellison, N.; Vitak, J. (2011). The "S" in Social Network Games: Initiating, maintaining, and enhancing relationships. System Sciences (HICSS), 2011 44th Hawaii International Conference on, 1-10. doi: 10.1109/HICSS.2011.400

Hancock, J. (2007). Digital deception: Why, when and how people lie online. In Oxford Handbook of Internet Psychology, eds. K. McKenna, T. Postmes, U. Reips \& A. N. Joinson. Oxford University Press.

Walther, J. B. (2007). Selective self-presentation in computer-mediated communication: Hyperpersonal dimensions of technology, language, and cognition. Computers in Human Behavior 23, 2538-2557

Wellman, B., Haase, A. Q., Witte, J., \& Hampton, K. (2001). Does the Internet increase, decrease or supplement social capital? Social networks, participation, and community commitment. American Behavioral Scientist 45(3), 436-455. 